\title{
Application To FRP Compound Material In Reinforcement And Repair Of Concrete Structures
}

\author{
Zhe Feng \\ Production and operation of the Daqing Oil Field Co
}

Keywords: FRP; Reinforcement; Concrete structure

\begin{abstract}
The bearing capacity of concrete beam can be improved considerably by FRP reinforcement. The engineering cases show that using FRP materials reinforce a balcony cantilever, and it can improve the concrete structure bearing capacity, and can prolong the service life in a certain extent, and can reduce the engineering cost. Thus, the process means can gain a great social economic benefit and can make a certain contribution.
\end{abstract}

\section{Preface}

In the dual role of the nature environment and the application environment, building's function is becoming weakened. So structural safety, durability and applicability will be reduced, and the service life of the structure is shortened in some extent. If demolition and rebuild are carried, it will cause economic waste. So the buildings need to be tested, repaired and reinforced. Using special materials for engineering reinforcement has caught the attention of the scholars. On the choice of reinforcement materials, FRP materials with light weight and high weight characteristics is researched and it is applied in the field of civil reinforcement engineering. FRP reinforcement technology research appeared in the 1980s [1], and it was first developed in Germany and Switzerland, then the United States, Japan, Canada and some European countries and regions of many universities, research institutions and materials manufacturers etc. Lots of fiber material is adopted to reinforce the concrete structure. FRP used to reinforce concrete structure technology is relatively common and has a good performance. Since the technology has wide applicability, good corrosion, durability and high efficiency, it has been more and more applied.

\section{FRP Material}

FRP (Fiber Reinforced Polymer) is made of polymer resin fiber[2]. Fiber is reinforced material, and it can stiffen effect, meantime the matrix material can bond and transfer the shear effect. The characteristics of FRP material is light weight, no corrosion, and a greater tensile strength. The tensile strength of FRP materials is obviously higher than that of steel. FRP material has good corrosion resistance, especially for materials such as acid, alkali and chlorine salt has a strong resistance to chemical corrosion.

FRP needs resin as matrix, and it is a bundle composite material which consists of the high strength and high modulus fiber. So far the fibers produced and applied are mainly glass fiber, carbon fiber and aramid fiber. Compared with other material mechanical properties, FRP material has a lot of advantages which is shown in Fig. 1. 


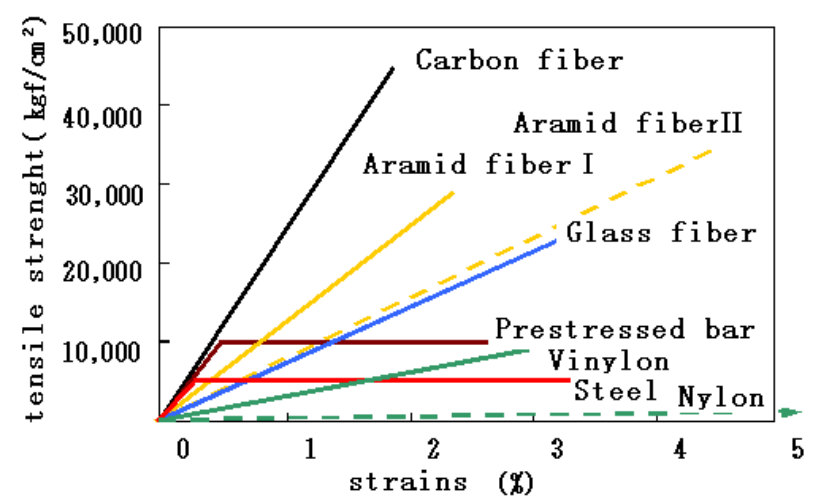

Fig. 1 Mechanical Performance Compare of FRP Materials with other Common Materials

\section{FRP Reinforcement Method}

The Embedded Reinforcement Method. In recent years, some researchers put forward the embedded reinforcement technology [3], and it is a very promising FRP reinforcement method. This method is to slot the components surface which is in the concrete protective layer, then to fill the groove with FRP bar or FRP lath, last to use the adhesive to combine FRP and the component. Using the good mechanical performance of FRP materials makes two kinds of material deform and work together. As a result, it can improve the anti-flexural and anti-shear capacity, and the component has the effect of reinforcement structures. The specific construction method is shown in fig. 2.
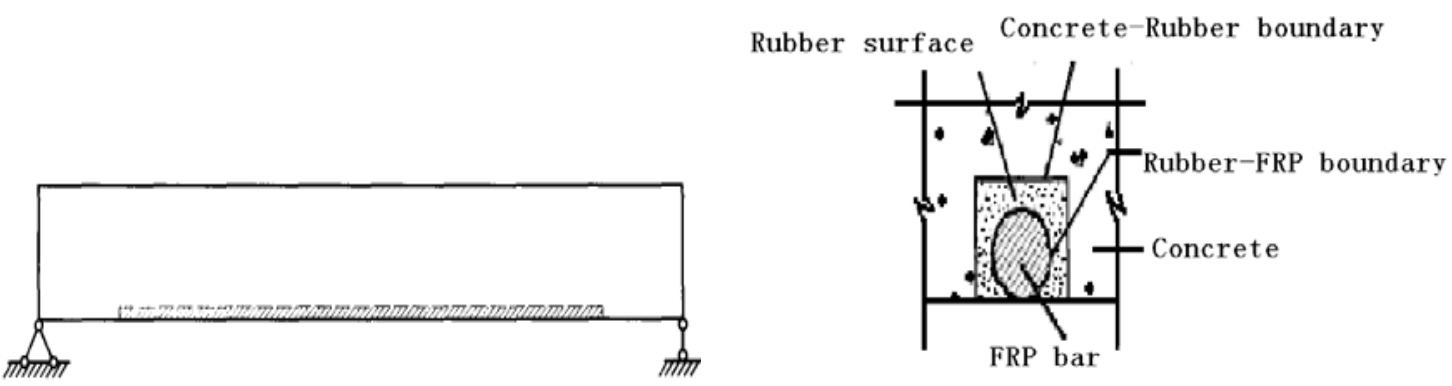

Fig. 2 Embedded Reinforcement Method Scheme

Using embedded reinforcement concrete member can make FRP materials be embedded in the concrete cover to avoid friction and collision accident load damage. The means is applicable to the reinforcement of bridge panel and continuous beam negative moment area. Increasing the bonding surface of FRP material can improve the utilization rate of FRP, and can reduce the workload of concrete component surface treatment in a certain extent, and can improve the work efficiency, and can facilitate to firm neighboring component each other. Use cement-based binder to replace epoxy resin, which can be applied to high temperature and high humidity in the concrete reinforcement engineering.

The embedded FRP reinforcement technology is more convenient, and don't need a lot of labor and large machinery, also don't need to occupy larger work space. But in order to ensure the test quality, construction should be carried out according to the following five steps:

(1) To slot on the surface structure according to design requirements;

(2) To remove the dust and the residue in the groove;

(3) To inject the binder in the groove to the half height;

(4) To put FRP bar or FRP lath into the groove and to press lightly, and to continue to inject the resin into the groove to the full;

(5) After the binder solidifying, the surface needs to be processed.

Reinforcement Methods With External Bonding. Using special binder use fiber cloth or sheet to paste on the concrete component surface, which method is called the reinforcement method with external bonding ${ }^{[3]}$. It is shown in fig. 3. In the late $80 \mathrm{~s}$, to study the stick steel reinforcement 
method, it appears a new thought that using the fiber reinforced material to reinforce the concrete structure instead of steel plate. After improving the technology, FRP material adopted in the reinforcement is mostly carbon fiber cloth material, and practical application becomes more and more popular.

Reinforcement methods with external bonding are easy to use, and have high efficiency, and don't need large construction machines and tools etc. Carbon fiber pre-dip material used in repair reinforcement is a one-way or two-way cloth fabric, and its width size mainly has $20 \mathrm{~cm}, 30 \mathrm{~cm}, 50$ $\mathrm{cm}$, even $100 \mathrm{~cm}$, and its length is $50 \sim 100 \mathrm{~m} /$ roll. In practice, the cloth can be cut any size with scissors or knife according to the real value, and construction is reliable. FRP pre-dip material is flexible, even though the reinforcement structure surface is not very smooth, and it also can guarantee effective paste rate nearly $100 \%$. If found the local surface of the reinforced component beam has bubble after solidify, also easy to repair, as long as the resin injection syringes with resin into the bubble to keep air out.

Because FRP material has good corrosion resistance, using stick approach for reinforced structures component don't need regular maintenance. The maintenance cost can be saved. FRP materials can play a role to protect the internal concrete structure to achieve the double repair concrete purpose.

The quality of FRP sheet external bonded is important for the reinforcement effect, so the construction should ensure the quality strictly. The concrete construction process is as follows:

(1)To process the base; (2)To coat the bottom rubber; (3)To level; (4)To bond; (5)To protect.

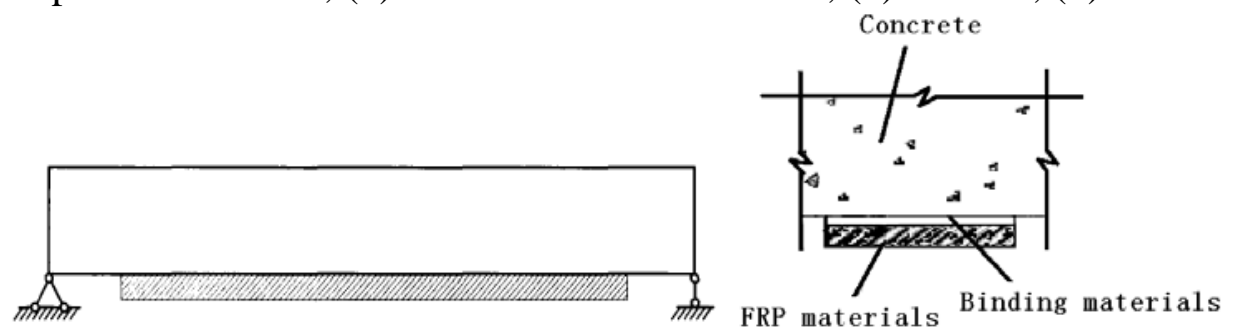

Fig. 3 Overlay Reinforcement Method Scheme

\section{Analyze and Calculate Reinforcement Theory}

(1)FRP net fiber tensile stress:

$$
\begin{aligned}
& f_{f u, f i b e r}=\frac{\bar{P}_{u}}{A_{f, \text { fiber }}}: \quad A_{f, \text { fiber }}=n t_{f, \text { fiber }} w_{f} \text { (Net fiber area); } \\
& \bar{p}_{u}=\frac{f_{f u, f i b e r} A_{f, \text { fiber }}}{w_{f}} \bar{p}_{u}=\frac{f_{f u, f i b e r} A_{f, \text { fiber }}}{w_{f}} \ldots \text { The stress value of unit width in FRP system }
\end{aligned}
$$

(2)The stress value of unit width of FRP system

$$
\begin{gathered}
\bar{p}_{u}=\frac{f_{f u, l a \min a t e} A_{f, l a \text { minate }}}{w_{f}}: A_{f, l a \text { min ate }}=t_{f, l a \text { min ate }} w_{f} \text { Total area of the sheet } \\
f_{f u, l a \text { min ate }}=\frac{\bar{P}_{u}}{A_{f, l a \text { min ate }}} \ldots \text { The tensile stress of total area in FRP system }
\end{gathered}
$$

(3)The main computational steps

I. Preliminary estimates

$f_{f u}=C_{E} f_{f u}^{*}, \varepsilon_{f u}=C_{E} \varepsilon_{f u}^{*} \quad$ To calculate material performance

$E_{c}=57,000 \sqrt{f_{c}^{\prime}} \quad$ The concrete performance

$\rho_{s} \equiv \frac{A_{s}}{b d}, \quad n_{s} \equiv \frac{E_{s}}{E_{c}} \quad$ The ordinary steel bar performance 
$A_{f}=n t_{f} w_{f}, \quad \rho_{f} \equiv \frac{A_{f}}{b d}, \quad n_{f} \equiv \frac{E_{f}}{E_{c}} \quad$ The external paste FRP performance

$\varepsilon_{b i}=\frac{M_{D L}(h-k d)}{I_{c r} E_{c}} \quad$ To determine the beam bottom strain state

$\kappa_{m}=1-\frac{n E_{f} t_{f}}{2,400,000} \quad$ To determine the paste coefficient of the FRP system

$c=0.20 d$ To estimate the neutral axis height c (Estimate neutral axis height reasonable preliminary value to be $0.20 \mathrm{~d}$, after check the balance equation, then adjust the c value)

$\varepsilon_{f e}=0.003\left(\frac{h-c}{c}\right)-\varepsilon_{b i} \leq \kappa_{m} \varepsilon_{f u} \quad$ To calculate the strain value of FRP

II. Calculate the stress, internal force and the balance equation

$\varepsilon_{s}=\left(\varepsilon_{f e}+\varepsilon_{b i}\right)\left(\frac{d-c}{h-c}\right) \quad$ To calculate ordinary steel bar strain (ordinary reinforced strain can be calculated using similar triangles)

$\mathrm{f}_{\mathrm{s}}=E_{s} \varepsilon_{s} \leq f_{y}, \quad f_{f e}=E_{f} \varepsilon_{f e}$

To calculate the stress of the steel bar and FRP $c=\frac{A_{s} f_{s}+A_{f} f_{f e}}{\gamma f_{c}^{\prime} \beta_{1} b} \quad$ To calculate internal force, to check balance equation, and to adjust c value until equilibrium equation satisfied

$\phi M_{n}=\phi\left[A_{s} f_{s}\left(d-\frac{\beta_{1} c}{2}\right)+\psi A_{f} f_{f e}\left(h-\frac{\beta_{1} c}{2}\right)\right]$ To calculate the bending strength cross section

III. To check the stress

Calculate the stress value, and ensure less than the limit value of formula recommended $f_{s, s} \leq 0.80 f_{y}$

$f_{s, s}=\frac{\left|M_{s}+\varepsilon_{b i} A_{f} E_{f}(h-k d / 3)\right|(d-k d) E_{s}}{A_{s} E_{s}(d-k d / 3)(d-k d)+A_{f} E_{f}(h-k d / 3)(h-k d)} \quad$ To calculate the stress value

Among them: Calculate the bending moment diagram area sum of the first transition section to crack the elasticity height of the neutral axis. Reinforced rectangular beam without compress can be simplified as follows:

$k=\sqrt{\left(\rho_{s} n_{s}+\rho_{f} n_{f}\right)^{2}+2\left(\rho_{s} n_{s}+\rho_{f} n_{f}\left(\frac{h}{d}\right)\right)}-\left(\rho_{s} n_{s}+\rho_{f} n_{f}\right)$

To calculate the Stress value of FRP $f_{f, s} \leq 0.55 f_{f u}$

$$
f_{f, s}=f_{s, s}\left(\frac{E_{f}}{E_{s}}\right)\left(\frac{h-k d}{d-k d}\right)-\varepsilon_{b i} E_{f}
$$

\section{An Engineering Example}

Rainbow home construction site is located in Daqing city, and the main body structure uses brick. In the process of construction, the $\Phi 8$ cantilever beam stirrups of the basic ring beam and a layer balcony bottom plate are found they can't be elongated to meet the requirements. According to the characteristics of the structure force, the steel bar can't guarantee it has no affect the ductility of the cantilever beam. Therefore, a suggestion given is to reinforce the stirrups. Concrete operation is as follow: Paste ring carbon fiber sheet on outer wall of the cantilever beam root facing the end. Sheet width is $100 \mathrm{~mm}$. Spacing is $250 \mathrm{~mm}$. Each beam pastes three. Lap position is at the top of the beam. Lap length is $150 \mathrm{~mm}$. Carbon fiber uses one-way grid cloth, and fabric weight is $200 \mathrm{~g} / \mathrm{m}^{2}$. Four corners of the beam are grinded fillet $15 \mathrm{~mm}$. The other construction procedures are needed to meet the relevant standards ${ }^{[4][5]}$. After the above methods treatment, that the low beam ductility of 
the structure damage can be avoided.

The Concrete Construction Process.To determine the position of construction $\rightarrow \rightarrow$ Basic level processing $\rightarrow \rightarrow$ To cut carbon fiber cloth according to the size $\rightarrow \rightarrow$ To make up adhesive glue $\rightarrow \rightarrow$ To past carbon fiber cloth $\rightarrow \rightarrow$ To roll and to paste firmly $\rightarrow \rightarrow$ To complete the construction.

Concrete Construction Methods And Construction Measures. I.To determine the position of construction:

According to the design requirements, line located in the construction site. In each cantilever beam end there are three width $100 \mathrm{~mm}$ and space $250 \mathrm{~mm}$ rings. For the other that can't form ring, it is grinded $100 \times 700 \mathrm{~mm}$ strip at the bottom along beam under the plate and it is pasted two layering to ensure the reinforcement quality.

II.Basic level processing:

(1)First use angle grinder machine to level the concrete bonding surface, and grind four corners fillet $15 \mathrm{~mm}$.

(2)Use the brush or the dryer to clear the concrete surface's dust and loose material, and make sure the surface is dry.

(3)Use cotton yarn with acetone or alcohol to wipe the concrete surface in order to remove the surface organic matter.

III.To coat the bottom glue:

(1)To confirm the construction environment

Because high moisture causes the bottom glue and moisture chemical reaction, it interferes the adhesive curing process. Pasting base should be without steam condensation to ensure construction quality.

(2)To choose bottom rubber material to coat the bottom glue.

Adhesive glue is divided into two groups, party A and B are mixed together according to the instructions, and the mixture is stirred until even color. Use container clean without oil for glue preparation, and avoid mixing with water into the container in order to prevent adhesive from lowing performance. Glue prepared coats evenly on the base with a brush.

IV. To cut the carbon fiber cloth:

According to the size of the design, use scissors or sharp knife to cut carbon fiber cloth. The carbon fiber cloth is rolled into a cylinder to avoid folding.

$\mathrm{V}$. To paste the carbon fiber cloth:

(1)After bottom glue coated has dried for about one hour to reach dry, stick up the carbon fiber cloth on the paste adhesive. Paste carbon FRP to make closed hoop as ring-shaped immediately. For the other can't be closed hoop, after sticking the U-shaped hoop, stick a layering at the bottom of the plate. Plastic roller rolls along the wire in the carbon fiber direction carefully until the binder will be fully saturated carbon fiber cloth. And then coat a layer adhesive evenly on the carbon fiber cloth.

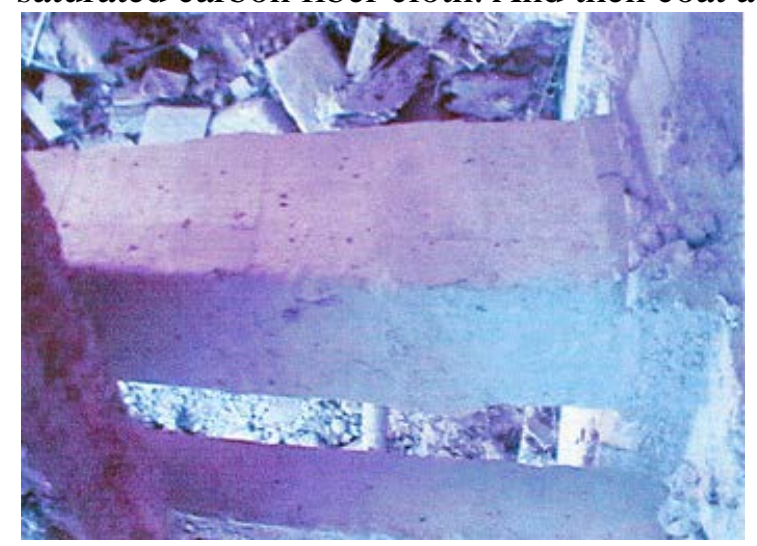

Fig.4 Before Reinforcement Concrete Beam

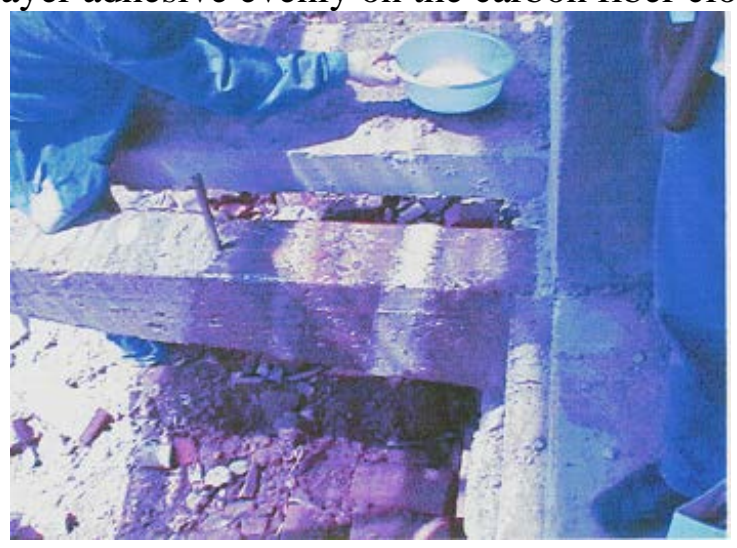

Fig.5 Before Reinforcement Preparing 


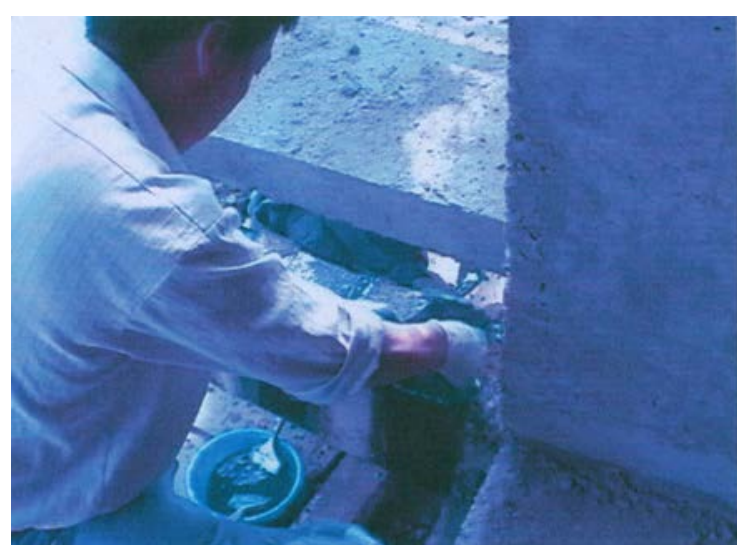

Fig.6 Field Construction Scheme

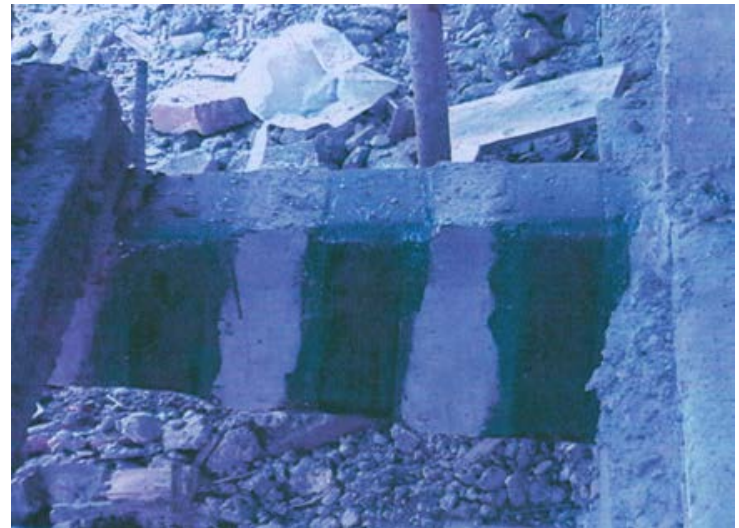

Fig.7 After Reinforcement Concrete Beam

(2)After having pasted the carbon fiber cloth paste, cast a layer of dry sand immediately on the carbon fiber cloth if the protection is needed to form the plastering protection.

\section{Conclusion}

It is proved through the engineering example that using FRP reinforced concrete structures can prolong the service life of concrete structures in a certain extent, and can reduce the engineering cost, and can obtain good social and economic benefits.

\section{Reference}

[1] Jiang Jianjing , Cui Jinghao, etc. Accident Analysis and Treatment of the Building Engineering[M]. Beijing: China Architecture Building Press, 2004(in Chinese)

[2] GB50367-2006, Code for Design of Concrete Structures Strengthening[S] (in Chinese)

[3] CECS 25:90.Technical Specification for Design of Concrete Structures Strengthening [S] (in Chinese)

[4] Wu Yong-he Anti-seismic Strengthening Design of An Apartment House[J]. Earthquake Resistant Engineering and Retrofitting. 2009.4 82 85.

[5] Chen An-pin.The Engineer Application Example of The House Reconstructed and Reinforced[J] Earthquake Resistant Engineering and Retrofitting, 2009.4: 79 95.

[6] Min-feng Li, Guo-fang Jin etc. Simplified calculation analysis of FRP reinforced concrete beam deformation[J]. Journal of sichuan building science, 2011, 11:87-90. (in Chinese)

[7] Tan Shuhua, Jiang Yu. FRP reinforced masonry structure technology study [J]. Journal of liaoning building, 2011 01:38 and 39. (in Chinese)

[8] Zan ShengFu. The research progress of FRP in civil engineering structure reinforcement application [J]. China water transport (second half), 2014,01:377-378. 